\title{
Stress modulates the use of spatial versus stimulus-response learning strategies in humans
}

\author{
Lars Schwabe, ${ }^{1,4}$ Melly S. Oitzl, ${ }^{3}$ Christine Philippsen, ${ }^{1}$ Steffen Richter, ${ }^{1}$ \\ Andreas Bohringer, ${ }^{1}$ Werner Wippich, ${ }^{2}$ and Hartmut Schachinger ${ }^{1}$ \\ ${ }^{1}$ Division of Clinical Physiology, Institute of Psychobiology, University of Trier, 54290-Trier, Germany; ${ }^{2}$ Division of Cognitive \\ Psychology, University of Trier, 54296-Trier, Germany; ${ }^{3}$ Division of Medical Pharmacology, Leiden/Amsterdam Center for Drug \\ Research, University of Leiden, 2300 RA-Leiden, The Netherlands
}

\begin{abstract}
Animal studies provided evidence that stress modulates multiple memory systems, favoring caudate nucleus-based "habit" memory over hippocampus-based "cognitive" memory. However, effects of stress on learning strategy and memory consolidation were not differentiated. We specifically address the effects of psychosocial stress on the applied learning strategy in humans. We designed a spatial learning task that allowed differentiating spatial from stimulus-response learning strategies during acquisition. In 13 subsequent trials, participants (88 male and female students) had to locate a "win" card out of four placed at a fixed location in a 3D model of a room. Relocating one cue in the last trial allowed inferring the applied learning strategy. Half of them participated first in the "Trier Social Stress Test." Salivary cortisol and heart rate measurements were taken. Stressed participants used a stimulus-response strategy significantly more often than controls. Subsequent verbal report revealed that spatial learners had a more complete awareness of response options than stimulus-response learners. Importantly, learning performance was not affected by stress. Taken together, stress prior to learning facilitated simple stimulus-response learning strategies in humans-at the expense of a more cognitive learning strategy. Depending on the context, we consider this as an adaptive response.
\end{abstract}

Memory consists of multiple anatomically and functionally distinct systems (Squire 1994; Gabrieli 1998; Eichenbaum and Cohen 2001; White and McDonald 2002). Evidence supporting this view comes from animal studies using brain lesion (Packard et al. 1989; Kesner et al. 1993; McDonald and White 1994; Packard and Teather 1998; Teng et al. 2000) or stimulation techniques (Packard et al. 1994; Packard 1999) as well as from human studies investigating brain-damaged patients (Haist et al. 1991; Bechara et al. 1995; Knowlton et al. 1996) or using functional neuroimaging (Iaria et al. 2003; Bohbot et al. 2004). Two systems received special attention in the multiple memory systems literature: one based on the hippocampus and adjacent cortices and one depending on the basal ganglia, specifically the caudate nucleus.

Mishkin and Petri (1984) suggested that the caudate nucleus mediates a "less cognitive, more rigid" form of memory termed "stimulus-response" or "habit" learning (for reviews of the cognitive dorsal striatum functions, see White 1997; Packard and Knowlton 2002). Since O'Keefe and Nadel's (1978) “cognitive map" theory, the hippocampus has often been associated with spatial memory (Nadel 1991; Maguire et al. 1998; Burgess et al. 2002). White and McDonald (2002) included the amygdala and proposed that the memory systems work in parallel. Packard and Teather (1998) demonstrated an improved memory performance in a hippocampus-dependent as well as in a caudate nucleusdependent task after amphetamine injections into the amygdala, suggesting that the amygdala exerts a modulatory influence on both "habit" and "cognitive" memory.

It is well known that stress affects memory: Facilitating as well as impairing influences are described. How stress within the context of a learning experience induces focused attention and

\section{${ }^{4}$ Corresponding author.}

E-mail schw1309@uni-trier.de; fax 49-651-201-3737.

Article is online at http://www.learnmem.org/cgi/doi/10.1101//m.435807. improves memory of relevant information, has been approached in various ways (for review, see Joels et al. 2006). Kim et al. (2001) suggested stress as a critical factor modulating the use of memory systems. In their study, rats received 60 tail shocks within 1 h 30 min before training in a Morris water maze to find a submerged platform, which location was marked by a pole. One day later, the platform and pole were relocated. Swimming to the training location of the platform was interpreted as a hippocampus-based spatial strategy. Swimming to the pole in the new location was seen as a caudate nucleus-based stimulus-response strategy. All nonshocked rats used a spatial strategy, whereas half of the stressed rats used a stimulus-response strategy. Functionality of the amygdala changed the performance as in the same study, rats with bilateral lesions of the amygdala showed stress-independent spatial strategies. Similarly, Packard and Wingard (2004) found that injections of anxiogenic drugs into the amygdala or periphery modulated the use of memory systems in a way that favors caudate-based stimulus-response learning over hippocampusbased spatial learning. Thus, being either stressed or anxious prior to learning will affect the use of multiple memory systems. It is important to note that performance expressed as latencies or distance to locate the platform were comparable, but strategies were not assessed during acquisition. Interestingly, in both studies, the effect of stress on memory systems was deduced from the rat's performance in the retention test $24 \mathrm{~h}$ later. Because the different memory systems are able to solve the task, a behavioral deficit can only be detected when the test situation includes competing responses (McDonald and White 1994; Packard et al. 1994; Packard and Wingard 2004).

The present study was designed to examine in humans the theory that stress affects the use of memory systems, expressed in distinct learning strategies. We hypothesized that previous psychosocial stress will result in the use of caudate nucleusdependent stimulus-response (habit) at the expense of hippo- 
A

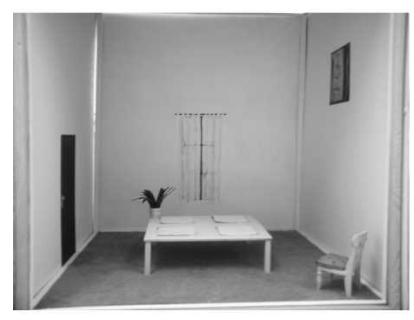

C

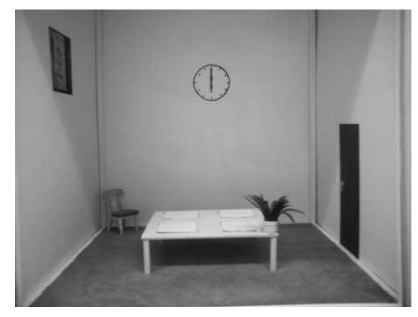

D

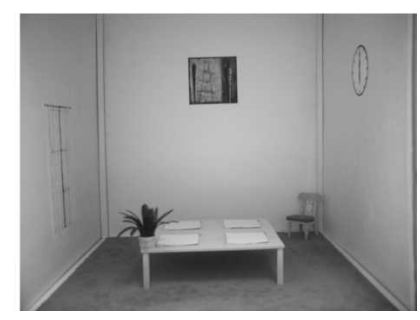

E

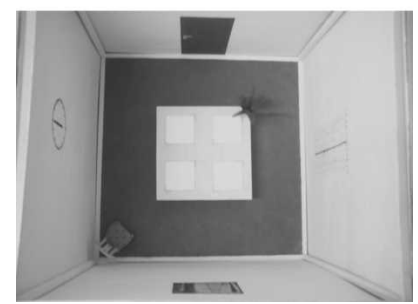

Figure 1. $3 \mathrm{D}$ model of the room (size $50 \times 50 \times 50 \mathrm{~cm}$ ) with removable walls: viewing angle $(A)$ the clock, $(B)$ the picture, $(C)$ the window, $(D)$ the door, and $(E)$ from above. The cards are placed up-side down on the table. The plant is at the same location on the table for 12 trials and relocated in trial 13 .

campus-dependent (cognitive) learning strategies. After participants were exposed to psychosocial stress (public speech and mental arithmetic in the Trier Social Stress Test, TSST) or a nonarousing control manipulation, they performed a newly developed spatial learning task. This task allowed differentiating spatial from stimulus-response learning strategies. Participants were presented a 3D model of a room (see Fig. 1) and asked to find a "win" card out of four cards. The task shares its central characteristics with the well-known spatial learning task for animals, the Morris water maze. The setup of the task was tested in a pilot study. The learning strategy used was derived from the actual performance of the participants as well as their verbal report. Heart rate measurements and salivary cortisol samples were taken at several time points to verify the efficacy of the stress procedure.

\section{Results}

\section{Performance in the learning task and verbal reports}

\section{Performance in the learning task}

Prior social stress affected the subsequent use of learning strategies (Fig. 2). A $\chi^{2}$-test computed on the strategies of the stressed and control participants that had learned the position of the win-card revealed a significant effect of experimental condition on the used learning strategy $\left[\chi_{(1)}^{2}=7.11 ; P<0.005\right]$. Only five participants of the stress group used a spatial strategy, 34 a stimu- lus-response strategy, and five did not acquire the task. In the control group, 15 participants used a spatial strategy, 23 a stimulus-response strategy, and six did not learn to find the win-card. Stress and control groups had a comparable learning performance [learning curve-speed of acquisition: $t_{(75)}=0.35 ; P=0.73$; learning gradient: Kaplan-Meier; $\left.\log \operatorname{rank} \chi_{(1)}^{2}=0.09 ; P=0.77\right]$ (data not shown). The learning performance of spatial and stimulus-response learners was comparable as well (Fig. 3) [learning curve-speed of acquisition: $t_{(75)}=0.56 ; P=0.58$; and learning gradient: Kaplan-Meier: $\log \operatorname{rank} \chi_{(1)}^{2}=1.04 ; P=0.31$.

\section{Verbal reports}

The report of spatial and stimulus-response learners about the use of strategies differed significantly $\left[\chi_{(3)}^{2}=47.75 ; P<0.001\right]$. All spatial learners ( $n=20$; independent of prior TSST) reported the use of a spatial strategy; 17 out of 20 (85\%) were also aware of the plant as a potential cue for the location of the win-card. All 57 stimulus-response learners reported the use of the stimulusresponse strategy, that is, plant is the cue for the location of the win-card; however, only nine of them (16\%) suggested a spatial option.

The certainty of their decision was neither affected by prior stress, nor related to the strategy used [Kruskal-Wallis $\chi_{(3)}^{2}=2.09$; $P=0.55]$ : stressed participants using a stimulus-response strategy or a spatial strategy (\% certainty; mean \pm SEM: $67.3 \pm 4.0$ or $76.0 \pm 13.4$ ), control participants with a stimulus-response strategy or spatial strategy (mean \pm SD: $63.9 \pm 5.2 ; 61.5 \pm 5.6$ ).

\section{Autonomic and endocrine measurements}

Autonomic and endocrine measurements verified the stressinduction by the Trier Social Stress Test (TSST).

\section{Cardiovascular responses}

Table 1 shows that heart rate was significantly enhanced in response to the Trier Social Stress Test (TSST) compared to controls $\left[\mathrm{t}_{(79)}=3.82 ; P<0.001\right]$. Before and after the TSST, heart rate was comparable between groups. The root mean square successive differences of the interbeat interval (RMSSDibi) were significantly lower in stressed participants than in controls during the experimental manipulation $\left[t_{(79)}=1.90 ; P=0.03\right]$ (Table 1$)$. Interestingly, RMSSDibi values of the stress group were already

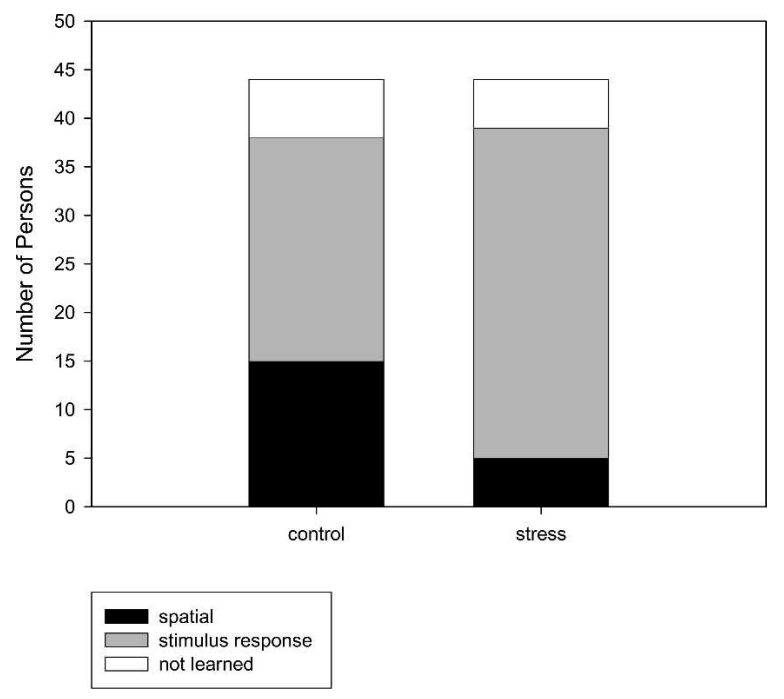

Figure 2. Number of participants of the stressed and control groups that were classified according to their learning performance to locate the win-card in trial 13 as spatial, stimulus-response, and non-learners.

\section{Learning \& Memory}




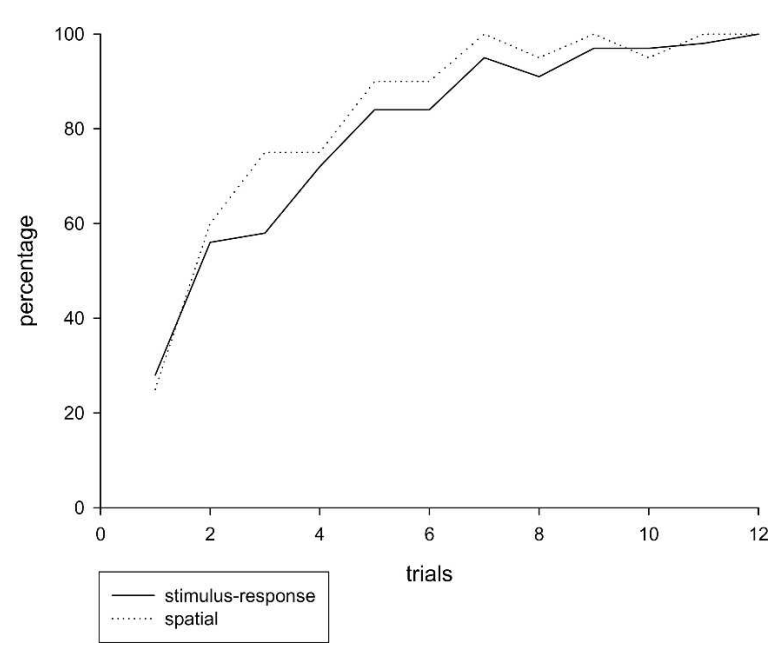

Figure 3. Learning performance: Percentage of participants who were identified as stimulus-response and spatial learners in trial 13 plotted against the number of trials they took to learn the location of the wincard. Note that the shape of the learning curve is independent of the strategy used.

lower before the TSST, indicating a fast parasympathetic response due to the announcement of the TSST. Moreover, all participants of the stress group $(n=44)$ reported during debriefing that they felt stressed during the TSST, while none of the controls $(n=44)$ described the control manipulation as stressful.

\section{Salivary cortisol responses}

Cortisol was increased in the participants of the TSST (group $F_{1,69}=13.423 ; P<0.0001$ ) (Fig. 4 A) with a different time course from the control group (time $F_{6,414}=29.098 ; P<0.0001$; interaction time $\times$ group $\left.F_{6,414}=10.235 ; P<0.0001\right)$. The cortisol levels of male and female participants did not differ within or between groups. Inspection of individual data revealed a subgroup of 10 (out of 36) "cortisol non-responders" in the stressed participants. Characterization of these subtypes are based on cortisol levels $>3$ $\mathrm{nmol} / \mathrm{L}$ at $20 \mathrm{~min}$ after the TSST. Stress and control groups differed significantly in (1) the number of cortisol responders and cortisol non-responders [cortisol $>3 \mathrm{nmol} / \mathrm{L}$ vs. cortisol $<3$ nmol/L in stressed group: $n=26$ vs. $n=10$; control: $n=7$ vs. $\left.n=29 ; \chi_{(1)}^{2}=20.20 ; P<0.001\right]$ and (2) the course of cortisol levels over time. In the control group, seven participants had a continuously elevated cortisol level $>3 \mathrm{nmol}$ from the first sample onward, but no salivary cortisol peak response. The time course of high cortisol of stressed and nonstressed participants differed significantly $\left(F_{6,185}=2.113 ; P<0.05\right)$, while it was comparable between the participants of both groups with low cortisol (see Fig. 4B,C).

Cortisol responders and cortisol non-responders of the stress group had comparably increased heart rates $(\mathrm{U}=98.5 ; P=0.78)$ and comparably reduced RMSSDibi $(\mathrm{U}=96.5 ; P=0.72)$; autonomic measures of the control group did not change over time (before, during, and after control manipulation) and were independent of their level of salivary cortisol (heart rate: $\mathrm{U}=65.0$; $P=0.18$; RMSSDibi: $\mathrm{U}=85.5 ; P=0.61$ ).

\section{Relationship between learning strategies, autonomic and endocrine activity} Independent of the experimental condition, the learning strategy used was correlated neither with heart rate $(r=0.06 ; P=0.62)$ nor with RMSSDibi $(r=0.01 ; P=0.92)$. There was also no significant correlation between heart rate and learning speed $(r=0.06$; $P=0.60)$, certainty $(r=0.02 ; P=0.86)$, or the number of possible strategies mentioned $(r=0.11 ; P=0.35)$. Similarly, the RMSSDibi was correlated neither with learning speed $(r=0.14 ; P=0.24)$ nor with certainty $(r=0.16 ; P=0.19)$, nor with the number of possible strategies mentioned $(r=0.06 ; P=0.60)$.

Independent of the experimental condition, there was no significant correlation between salivary cortisol 20 min after the end of the TSST and control manipulation and the learning strategy used $(r=0.12 ; P=0.30)$. However, as shown in Table 2 , the mean salivary cortisol levels 20 and $30 \mathrm{~min}$ after the end of the TSST, that is, during the learning task, tended to be higher in stimulus-response than in spatial learners-both in the stress (Cortisol +20: $U=38.5 ; P<0.05$; Cortisol +30 : $U=41.0 ; P<0.06$ ) and the control group (Cortisol +20 : $U=73.0 ; P=0.12$; Cortisol +30: $\mathrm{U}=77.5 ; P=0.17$ ).

Salivary cortisol did not correlate significantly with learning speed $(r=0.17 ; P=0.15)$, certainty $(r=0.10 ; P=0.41)$, or the number of possible strategies mentioned $(r=0.18 ; P=0.13)$.

There was no effect of sex on the strategy used $\left[\chi_{(1)}^{2}=1.15\right.$; $P=0.29]$. Furthermore, performance in trial 13 was independent of the side from which participants looked into the room, in the stress $\left[\chi_{(6)}^{2}=1.75 ; P=0.94\right]$ as well as in the control condition $\left[\chi^{2}{ }_{(9)}=12.83 ; P=0.17\right]$.

\section{Discussion}

We developed a spatial learning task to translate the findings of an animal study on multiple memory systems (Kim et al. 2001) to humans. Our results demonstrate that psychosocial stress prior to learning reduces the use of subsequent spatial learning strategies in favor of stimulus-response learning strategies.

These findings are generally in line with the results of an animal study (Kim et al. 2001) showing that prior footshock stress enhances dorsal striatum-dependent "habit" memory at the expense of hippocampus-dependent "cognitive" memory when tested $24 \mathrm{~h}$ later. Half of their stressed and all control rats displayed a spatial response in the memory test. In our study, stimulus-response learning was expressed by $77 \%$ of the stressed and $52 \%$ of the control participants, leaving $11 \%$ spatial learning in the stressed and 35\% in controls. This different prevalence of spatial and stimulus-response learners in both studies (1) might be a reflection of task characteristics or species, and the fact that (2) the Kim study based their findings on the performance $24 \mathrm{~h}$ after training. Then, stress effects on learning strategies might be obscured by stress effects on memory consolidation. In our study, we focused solely on the learning strategy, with little impact of memory consolidation processes during the rather short learning period to be expected. Other aspects of the learning process like efficacy and speed of learning, expressed by latencies to platform,

Table 1. Heart rates and RMSSDibi before, during, and after the experimental manipulation

\begin{tabular}{lcclll}
\hline & \multicolumn{2}{c}{ TSST } & & \multicolumn{2}{c}{ Control manipulation } \\
\cline { 2 - 3 } \cline { 6 - 7 } & Heart rate & RMSSDibi & & Heart rate & RMSSDibi \\
\hline Before & $89.7 \pm 2.1$ & $24.7 \pm 1.8$ & & $89.1 \pm 2.1$ & $29.3 \pm 3.0$ \\
During & $107.4 \pm 3.2^{\text {a,b }}$ & $24.6 \pm 2.0^{\text {a }}$ & & $93.5 \pm 2.0$ & $31.8 \pm 3.1$ \\
After & $91.8 \pm 2.2$ & $28.4 \pm 2.6$ & & $90.1 \pm 2.0$ & $27.7 \pm 2.6$ \\
\hline
\end{tabular}

Heart rate (beats per minute) and the root mean square successive difference of the interbeat interval (RMSSDibi) before, during, and after the Trier Social Stress Test (TSST) and the control manipulation. Increased heart rate and decreased RMSSDibi in the stress group underline the efficacy of the TSST. No change in these measures in the control group. Note the lower RMSSDibi of the stressed group before actually performing the TSST: These participants were informed about the TSST procedure. Data represent means \pm SEM.

$P<0.05$ abetween groups, ${ }^{b}$ within group. 
were comparable between the groups and thus, not affected by prior stress (Kim et al. 2001). We corroborate these findings as in our study, the number of "non-learners," the number of trials to the defined learning criterion, that is, learning speed, were also comparable between the nonstressed and stressed groups. Importantly, conscious report of spatial learners revealed that many of them were aware of two potential response options, the spatial
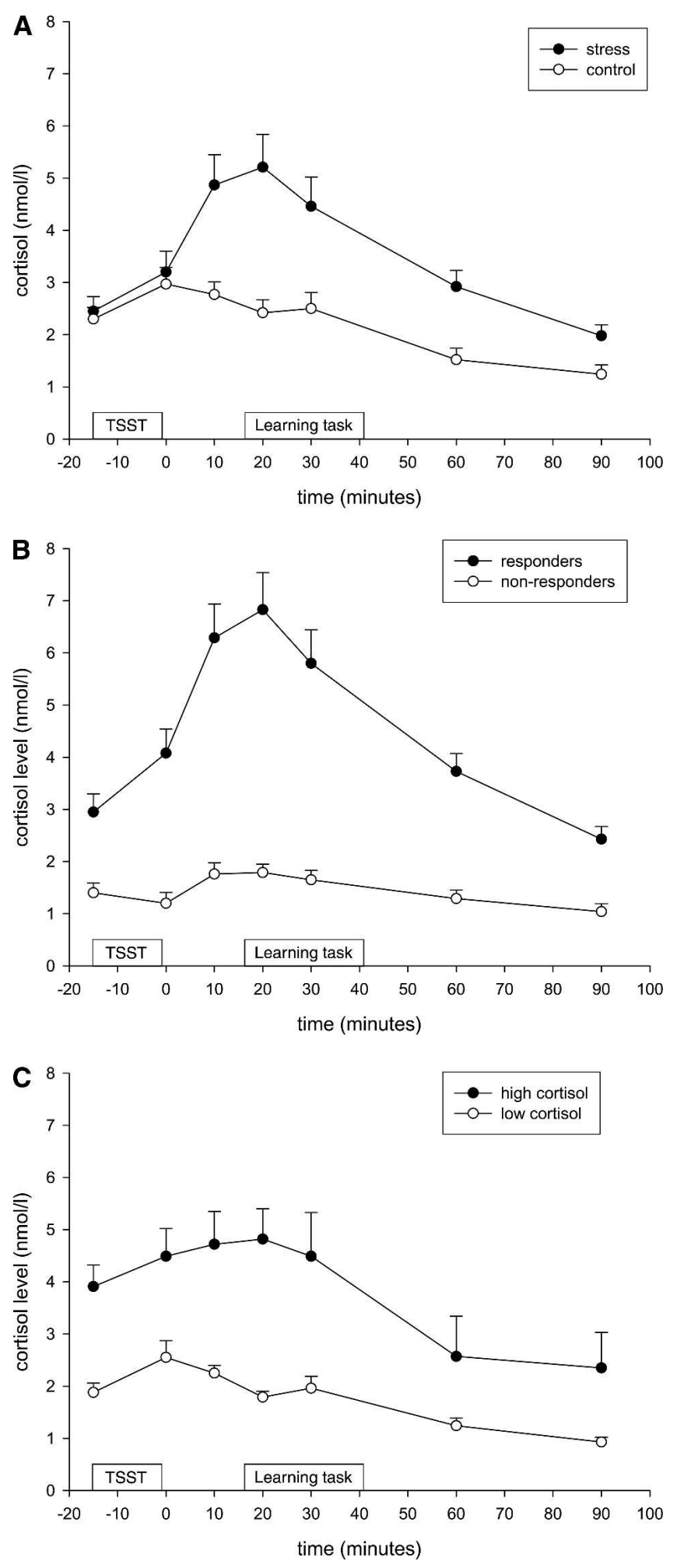

and stimulus-response options. Thus, spatial learning has to be considered as more elaborated. Concluding the comparison of the rat and human studies, we suggest that prior stress facilitates the use of a dorsal striatum-dependent "habit" learning strategy at the expense of a flexible, elaborated, "cognitive" way of learning based on medial temporal lobe structures.

Dorsal striatum-based and hippocampus-based memory systems have been shown to process information in parallel and simultaneously (White and McDonald 2002). While the relationship between the two memory systems is cooperative in some cases (McIntyre et al. 2003; Voermans et al. 2004), they compete in others (Matthews and Best 1995; Poldrack et al. 2001; Schroeder et al. 2002). Characteristics of the particular task (McDonald and White 1993; White and McDonald 2002) as well as the frequency of task performance (Packard 1999; Chang and Gold 2003) are decisive for the relative participation of memory systems. Moreover, White and McDonald (2002) extended these two parallel memory systems by a third, the amygdala. Packard and Wingard (2004) discuss the basolateral amygdala as modulator of the cognitive and habit memory processes mediated by the hippocampus and caudate nucleus, respectively. Indeed, also Kim and colleagues (2001) suggested a specific role of the amygdala. Bilateral lesions of the amygdala of rats block the impairing effect of stress on spatial memory and hippocampal long-term potentiation. There is an intriguing interaction between stress hormones (catecholamines and glucocorticoids), amygdala, and hippocampus (Roozendaal et al. 2006). The memory-enhancing effects of glucocorticoids administered immediately after learning are blocked by lesions of the basolateral amygdala. Whether stress acts either directly via the hippocampal or amygdala memory system, or by modulating influences via the amygdala, remains to be established. The findings of the present study reveal stress as an important factor affecting the use of multiple memory systems.

This raises the question why one system dominates behavior, if both systems are intact. It is evident that stressed participants more likely use less complex learning strategies: the use of the stimulus-response strategy was increased by $50 \%$. In stressful situations, attention has to be diverted, and fast reactions are required. Hesitation, delays, might endanger the organism. Stimulus-response learning is more rigid, and thus, reduces ambiguity and interfering conflict. It is cognitively less demanding than spatial learning, leaving more cognitive capacities for coping with the current stress and its consequences. Stimulusresponse learning may not depend on an explicit cognitive reflection, but rather a "habit" formation. It appears reasonable to assume that these are accessible more quickly than "cognitive" (e.g., spatial) memories. The lack of cognitive reflection inherent to stimulus-response learning and memory is paralleled by a relative insensitivity to situational changes that may prompt for change of behavior. This idea is in line with the concept of "bounded rationality" (Simon 1982; Gigerenzer and Goldstein

Figure 4. Salivary cortisol in nanomoles per liter (mean \pm SEM) was measured at several time points throughout the experiment. The boxes in the graph denote the time point and duration of the Trier Social Stress Test (TSST) and the control manipulation as well as the time point and duration of the spatial learning task. Comparison of $(A)$ stress group $(n=37)$ and control group $(n=35)$. Note that the learning task was presented during the period of high salivary cortisol of the stress group. Next, participants were subdivided into groups with cortisol $>3 \mathrm{nmol} / \mathrm{L}$ at $t=20 \mathrm{~min}$, that is, at the beginning of the learning task. This resulted in the following: (B) Subdivision of the stress group into participants who responded with increased salivary cortisol to the TSST $(n=26)$ versus participants who did not respond $(n=10)$. (C) Subdivision of the control group into participants with elevated cortisol (high cortisol group, $n=7$ ) versus lower cortisol $(n=29)$.

\section{Learning \& Memory}


Table 2. Cortisol during the spatial learning task

\begin{tabular}{|c|c|c|c|c|}
\hline & \multicolumn{2}{|c|}{ Stress } & \multicolumn{2}{|c|}{ Control } \\
\hline & Spatial & $\begin{array}{l}\text { Stimulus- } \\
\text { response }\end{array}$ & Spatial & $\begin{array}{l}\text { Stimulus- } \\
\text { response }\end{array}$ \\
\hline & $(n=5)$ & $(n=30)$ & $(n=11)$ & $(n=18)$ \\
\hline Cortisol 20 & $3.0 \pm 1.1$ & $5.6 \pm 0.7^{a, b}$ & $1.9 \pm 0.3$ & $2.4 \pm 0.4$ \\
\hline Cortisol 30 & $2.6 \pm 1.0$ & $4.8 \pm 0.6^{a}$ & $1.8 \pm 0.2$ & $2.8 \pm 0.5$ \\
\hline
\end{tabular}

Saliva cortisol in nanomoles per liter during the learning task, that is, 20 min (cortisol 20) and 30 min (cortisol 30) after the TSST or control manipulation. $n$ denotes the number of participants of the stress and control groups using the spatial or stimulus-response strategy with cortisol data at all time points. There is a trend that stimulus-response learners have a higher level of cortisol than spatial learners. Data represent means \pm SEM.

$P<0.05$ abetween stress and control groups, ${ }^{\mathrm{b}}$ between spatial and stimulus-response learners in the stress group.

1996) postulating that participants often use heuristics, that is, relatively simple rules, which lead to cognitive relief and correct decisions in many situations-but not in all. In general, the arguments above underline stimulus-response strategies as adaptive behavioral response to stress.

Next, we might question the general belief that explicit memory processes are excluded in the case of stimulus-response learning. After the last trial, participants were asked to memorize and report their strategy, and also other options they were aware of: a process involving explicitly accessible knowledge and memories. Spatial as well as stimulus-response learners reported full awareness of their strategy used, that is, they could explain their thoughts when they had to make the decision. Consistently, there are reports that caudate-based learning and memory also may include explicit processes. For example, Voermans et al. (2004) demonstrated that the explicit learning of a stimulusresponse association led to significant activity of the right caudate nucleus. Sprengelmeyer et al. (1995) indicated that impairments in declarative memory are associated with the severity of the damage to the caudate loop. Furthermore, Maddox and Ashby (2004) viewed the head of the caudate nucleus as part of an explicit category learning system. The access of stimulusresponse learners to declarative retrieval like spatial learners does not contradict the notion that the two learning strategies belong to distinct memory systems. It might be a human characteristic, not likely to be tested in nonprimates.

The spatial learning task we developed has some central characteristics in common with the main spatial navigation task used to test hippocampal functions in rodents (Morris water maze): fixed location of the goal, various starting points. Until trial 12, spatial as well as stimulus-response strategies were successful in solving the problem: to locate the win-card. In the last trial, a simple and unspectacular relocation of the cue (stimulus), the plant, allowed for assessment of the strategy used. In contrast, the strong aversive and life-threatening feature (drowning) that motivates the performance of rats is absent. Our task might even be considered as slightly appetitive because of the small monetary reward. On the other hand, we are confronted with a specific human response, namely, the expectation of good performance when participating in an experiment. We cannot exclude that this is also experienced as social pressure and thus, context-related stress.

Psychosocial stress as induced by the TSST results in the co-occurrence of endocrine and autonomic responses (Schommer et al. 2003). During the TSST, we observed in all participants significant increases in heart rate and decreases in the root mean square successive difference of the interbeat interval (RMSSDibi), indicative of a strong activation of the autonomic nervous system toward a more dominant sympathetic than parasympathetic control. After the TSST, these measures had returned to baseline. Salivary cortisol started to increase after the TSST and reached peak levels during the spatial learning task. Both, autonomic and endocrine activity of the majority of TSST participants followed the time course of stress-system activation, underlining the efficacy of the TSST as an inducer of psychosocial stress.

Basal and stress levels of cortisol vary across individuals. While the endocrine stress response of the majority of the TSST participants reached peak levels, others kept low cortisol levels throughout the study (here: 10 out of 36), often called cortisol non-responders. Some participants of the control group (seven out of 36) had elevated cortisol levels throughout the study, which might reflect a combination of an anticipatory elevated activity of the stress system and endocrine activation by social demands. In the stress group of the present study, two spatial learners were classified as cortisol responders, while three spatial learners had no cortisol response. Together with the data of spatial learners of the control group, spatial learning strategies are more likely in the face of low cortisol levels. The number of individuals, however, is too small to allow test statistics related to spatial and stimulus-response strategies.

Stress hormones like catecholamines and glucocorticoids are well known for their modulating action on memory consolidation and retrieval (for review, see Joels et al. 2006). Less is known of their impact on learning strategies, although some investigators indicate that glucocorticoids and stress prior to a memory task are associated with improved performance, implying beneficial effects on memory acquisition and encoding (Shors et al. 1992; Lupien et al. 2005). Most of the literature focuses on the impairing effect of stress and glucocorticoids on hippocampusdependent declarative memory (for review, see Lupien et al. 2005). The mechanism of action of stress hormones, particularly glucocorticosteroids, can explain how stress within the context of a learning experience induces focused attention and improves memory of relevant information (de Kloet et al. 1999; Joels et al. 2006). Glucocorticoids act via two receptors, the mineralo- (MR) and glucocorticoid receptors (GR) that are colocalized in limbic regions. Well known are the genomic actions via GR on memory consolidation. Novel is the discovery of a low-affinity, membrane-bound MR with non-genomic action (Karst et al. 2005). The role of MR in behavior in novel situations, a given strategy to explore the environment, has been postulated in animal studies (Oitzl and de Kloet 1992), and recently confirmed in mice with a specific forebrain knockout of the MR (Berger et al. 2006). At present, we can just speculate on the brain structures involved and molecular mechanism underlying the stress effect on learning strategies. Given the time course of cortisol levels during task performance, we might consider an involvement and nongenomic activity of brain MR in the modulation of strategies.

Nadel et al. (2002) suggested that stress impairs primarily the ability of the hippocampus to bind stimuli together as belonging to a specific context. Under stress "one loses the ability to use critical distinguishing information" (Nadel et al. 2002), a statement in line with the findings of the present study. The regulating mechanism is most likely a shift in dominance of memory systems.

Stress increased stimulus-response learning by almost $50 \%$ and reduced the use of spatial learning strategies by $66 \%$ compared to the control condition. Although stress and glucocorticoid effects have to be considered in the context of actual needs and the strategy seems to be adaptive at the very moment, the long-term consequences might be less so. 


\section{Materials and Methods}

\section{Pilot study}

Twenty-two students (nine females, 13 males; mean age: $24 \mathrm{yr}$; range: $19-34 \mathrm{yr}$ ) participated in a pilot study to test the setup of the learning task. The test apparatus is described below. Briefly, participants had to locate the win-card in 12 trials with fixed locations of the cues. In trial 13, a proximal cue was relocated, allowing the differentiation between stimulus-response and spatial learning strategies. After indicating the location of the wincard, interviews were taken. Eleven participants used a stimulusresponse strategy, seven used a spatial strategy, and four participants did not learn the task. The verbal reports revealed that spatial and stimulus-response learners differed with respect to their awareness of the two strategies $\left[\chi_{(1)}^{2}=7.90 ; P<0.005\right]$. While six out of seven (86\%) spatial learners reported that spatial and stimulus-response strategies are possible, only two out of 11 (18\%) stimulus-response learners did so. Spatial and stimulusresponse learners did not differ in their learning curves [KaplanMeier survival analysis; $\left.\log \operatorname{rank} \chi_{(1)}^{2}=0.46 ; P=0.50\right]$ or in their certainty of choice $\left[t_{(16)}=1.10 ; P=0.29\right]$. Performance of male and female participants was comparable $\left[\chi_{(1)}^{2}=0.51 ; P=0.47\right]$.

\section{Main study}

\section{Participants}

Eighty-eight (62 females, 26 males) students of the University of Trier in the age range of $19-35 \mathrm{yr}($ mean $=23.2 \mathrm{yr} ; \mathrm{SD}=3.14)$ agreed to participate in the investigation. All of them were medication free, and none of the participants showed evidence of drug abuse. They had to refrain from smoking, drinking caffeine, or having severe physical exercise on the test day. Experiments were performed in the afternoon between 14:00 and 16:00 h. Participants received a monetary incentive on completion of the experiment. Written informed consent was obtained from all participants.

\section{Experimental design}

Participants were pseudo-randomly assigned to the control and the psychosocial stress conditions. Sexes were counterbalanced with $n=31$ females and $n=13$ males per group. The time line of the experiment is shown in Figure 5.

\section{Psychosocial stress}

Psychosocial stress was induced by the Trier Social Stress Test (TSST). Participants have to give a free speech and perform mental arithmetic in front of an audience (Kirschbaum et al. 1993). As has been shown previously, this protocol leads to significant sympathetic and endocrine activation (Schommer et al. 2003), used as read-outs of the experienced stress. During the initial briefing, participants were told that they are going to participate in a psychosocial stress test and introduced to the task; 3 min was allowed to prepare a presentation in which they had to promote their candidacy for a job that was tailored to their interests and qualifications. The audience consisted of a male and a female, dressed in a white coat, sitting at a table opposite to the standing participant. To increase task engagement, participants were told that their talk will be audio- and videotaped for later analyses. After 5 min of job presentation, arithmetic had to be performed, also standing in front of the audience. Subtraction of 17 serially from 2023 had to be done as accurately and fast as possible. Upon a mistake, the participant had to stop and start again at 2023. The mental arithmetic task took 5 min. Participants were not told how long the TSST and its single elements would take.

In the control conditions, the participants stood quietly in the room for $3 \mathrm{~min}$. Thereafter, they read a standard text for 5 min, followed by reading of a standardized list of four-digit numbers for another $5 \mathrm{~min}$. No audience was present, and no videorecordings were taken.

During the 15-min break between the TSST/control manipulation and the spatial learning task, the participants remained in

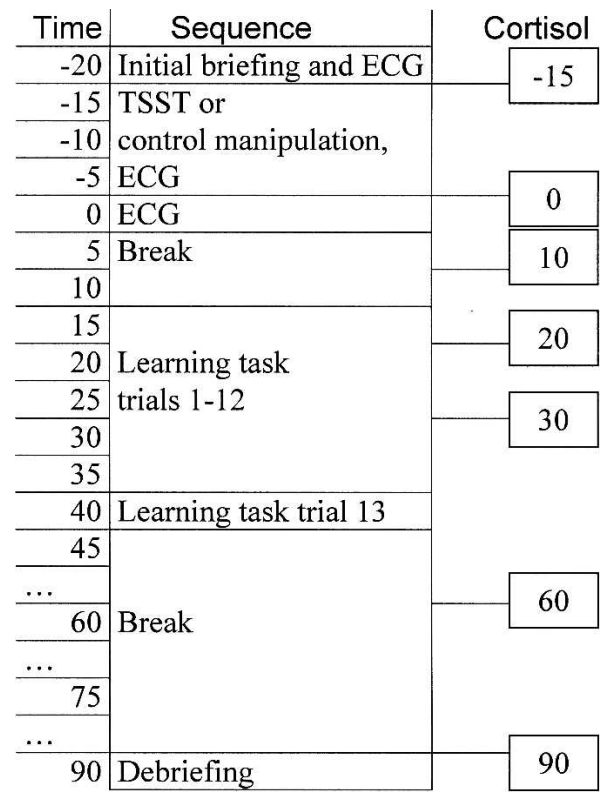

Figure 5. Schedule, listing the time and sequence of events during the experimental session. (Left column) Timeline in minutes; $t=0$ denotes the end of the Trier Social Stress Test (TSST) or control manipulation. (Middle section) Experimental procedure. (Right column) The time points of salivary cortisol collection.

a separate room, quietly reading, except when autonomic and endocrine measurements were taken.

To verify the efficacy of the TSST to induce stress compared with the control condition, heart rate was recorded for $5 \mathrm{~min}$ immediately before the TSST/control manipulations (pre-stress), during the $13 \mathrm{~min}$ of the TSST/control manipulations, and for 5 min immediately after the TSST/control manipulations. Moreover, saliva cortisol samples were collected immediately before the stress manipulation $(t=-15 \mathrm{~min})$; directly after the TSST $(t=0)$; as well as 10, 20, 30, 60, and 90 min after the end of the TSST/control manipulations.

Post hoc, we characterized subtypes of cortisol profiles; a cortisol level of at least $3 \mathrm{nmol} / \mathrm{L}$ at $t=20 \mathrm{~min}$ after the end of the TSST or control condition was used to subdivide participants into cortisol responders and cortisol non-responders, respectively.

\section{Spatial learning task}

\section{Apparatus}

Participants were presented a wooden 3D model of a room (box $50 \times 50 \times 50 \mathrm{~cm})($ Fig. $1 \mathrm{~A}-\mathrm{E})$. In the center of this room is a square table on which four identical cards (white side up) are placed, exactly in the middle of one of the four quadrants. There is a small plant in one of the corners of the table. Each wall contains one cue: door, window, picture, or clock. These cues are exactly in the middle of the walls. Therefore, a direct association of one of these cues to one of the four cards is excluded. A chair is placed in a corner of the room. All these symbols should allow spatial orientation. The box is revolvable; the walls can be removed.

\section{Procedure}

Fifteen minutes after the stress manipulation, participants were told that they would see a 3D model of a room, containing among other things four cards lying on a table. One of the four cards would be a "win-card" (word "win" written on the card), while the other three cards would be "no-win" cards (word "blank" written on the card). The backsides of the cards were white and visible. One wall of the box was removed. The partici-

\section{Learning \& Memory}


pant sat directly in front of the model and was asked to point with the finger at the card which he/she guessed to be the wincard. The experimenter turned this card in such a way that the subject saw the text on the card. In this way, participants received an immediate positive or negative feedback. The participant received a small monetary reward (50 Euro-cents) for locating the win-card. Thirteen trials were given. Eyes had to be closed between the trials. The experimenter turned the box, replaced one, and removed another wall after every trial (same sequence for all participants). In this way, each trial provided a different view into the same room, with all objects in a fixed position. In trials 1 to 12 , participants looked three times from each of the four sides into the room. The participants were not told that the win-card was at the same position in all trials.

In the course of 12 trials, the subject could acquire the position of the win-card either by learning that the win-card was always next to the plant (stimulus-response) or by learning the position of the win-card relative to other room cues (spatial). Since the probability is $25 \%$ to locate the correct card by chance, we used a strict criterion to define learning: The win-card had to be chosen in three consecutive trials without change in the following trials. The learning (acquisition) speed was set to this same trial. Performance in trial 13, the last trial, revealed the learning strategy. In this trial, the plant (stimulus) had been moved to another corner of the table. The use of a spatial strategy was accepted, if a participant pointed at the card in the quadrant in which the win-card had been located in trial 12. Choosing the card next to the plant was considered as a stimulus-response strategy.

To exclude the possibility that the decision in trial 13 might be influenced by the side from which participants look into the room, the latter was varied between participants.

\section{Verbal report}

After the participant had made the choice for location of the win-card in trial 13, but before receiving feedback, we asked him/ her (1) to describe the strategy used, (2) if there might be a reasonable alternative, and (3) to estimate the certainty of the decision on a scale from 0 to 100 , where 0 stands for "absolutely uncertain" and 100 for "absolutely certain."

\section{Cardiovascular analyses}

Heart rate was derived from a single standard lead II ECG configuration using a telemetric HP 78100A transmitter and HP 78101A receiver system (Hewlett Packard Corp.). ECG was sampled by $1 \mathrm{kHz}$ with 12-bit resolution. Beat detection was performed offline by WinCPRS (Absolute Aliens Oy) as was artifact control. The following parameters, which have been used successfully in stress research (Buchholz et al. 2003), were used: mean heart rate and the root mean square successive differences of the interbeat interval (RMSSDibi), the latter being a sensitive index of stress-induced vagal withdrawal.

Autonomic measurements were taken before, during, and at the end of the TSST or control manipulation.

\section{Collection of saliva and biochemical analyses}

Saliva samples were taken with a customary straw, put directly into standard Eppendorf tubes (1.5 mL; Eppendorf), stored at room temperature until completion of the session, and then kept at $-20^{\circ} \mathrm{C}$ until analysis. After thawing for biochemical analysis, the fraction of free cortisol in saliva (salivary cortisol) was determined using a time-resolved immunoassay with fluorometric detection, as described in detail elsewhere (Dressendorfer and Kirschbaum 1992).

\section{Statistical analysis}

We used the SPSS-statistical package (version 13.0; SPSS Inc.). If not indicated otherwise, two-tailed $P$-values are reported. Significance was accepted at $P<0.05$. Owing to technical failure, we lost some ECG data: baseline ECGs for three participants of the stress group and one of the control group; and stress ECGs and post-stress ECGs for three and two participants of the stress group, respectively. Furthermore, cortisol data were missing for eight participants of the stress and control groups.

\section{Acknowledgments}

This work was part of the Trier-Leiden IRTG program, supported by grants DFG GRK 1389/1 and NWO DN 95-420. Furthermore, this study was supported by the Forschungsfonds of the University of Trier. The participation of M.S.O. was supported by NWO grant 051.02 .10 . The participation of C.P. was supported by DFG grant SCHA12372-1.

\section{References}

Bechara, A., Tranel, D., Damasio, H., Adolphs, R., Rockland, C., and Damasio, A. 1995. Double dissociation of conditioning and declarative knowledge relative to the amygdala and hippocampus in humans. Science 269: 1115-1118.

Berger, S., Wolfer, D.P., Selbach, O., Alter, H., Erdmann, G., Reichardt, H.M., Chepkova, A.N., Welzl, H., Haas, H.L., Lipp, H.P., et al. 2006. Loss of the limbic mineralocorticoid receptor impairs behavioral plasticity. Proc. Natl. Acad. Sci. 103: 195-200.

Bohbot, V., Iaria, G., and Petrides, M. 2004. Hippocampal function and spatial memory: Evidence from functional neuroimaging in healthy participants and performance of patients with medial temporal lobe resections. Neuropsychology 18: 418-425.

Buchholz, K., Schächinger, H., Wagner, M., Sharma, A.M., and Deter, H.C. 2003. Reduced vagal activity in salt-sensitive participants during mental challenge. Am. J. Hypertens. 16: 531-536.

Burgess, N., Maguire, E.A., and O'Keefe, J. 2002. The human hippocampus and spatial and episodic memory. Neuron 35: 625-641.

Chang, Q. and Gold, P. 2003. Switching memory systems during learning: Changes in patterns of brain acetylcholine release in the hippocampus and striatum in rats. J. Neurosci. 23: 3001-3005.

de Kloet, E.R., Oitzl, M.S., and Joels, M. 1999. Stress and cognition: Are corticosteroids good or bad guys? Trends Neurosci. 22: 422-426.

Dressendorfer, R.A. and Kirschbaum, C. 1992. Synthesis of a cortisol-biotin conjugate and evaluation as a tracer in an immunoassay for salivary cortisol measurement. J. Steroid Biochem. 43: $683-692$.

Eichenbaum, H. and Cohen, N.J. 2001. From conditioning to conscious recollection: Memory systems of the brain. University Press, Oxford.

Gabrieli, J.D. 1998. Cognitive neuroscience of human memory. Annu. Rev. Psychol. 49: 87-115.

Gigerenzer, G. and Goldstein, D.G. 1996. Reasoning the fast and frugal way: Models of bounded rationality. Psychol. Rev. 103: 650-669.

Haist, F., Musen, G., and Squire, L.R. 1991. Intact priming of words and nonwords in amnesia. Psychobiology 19: 275-285.

Iaria, G., Petrides, M., Dagher, A., Pike, B., and Bohbot, V. 2003. Cognitive strategies dependent on the hippocampus and caudate nucleus in human navigation: Variability and change with practice. J. Neurosci. 23: 5945-5952.

Joels, M., Pu, Z., Wiegert, O., Oitzl, M.S., and Krugers, H.J. 2006. Learning under stress: How does it work? Trends Cogn. Sci. 10: $152-158$.

Karst, H., Berger, S., Turiault, M., Tronche, F., Schutz, G., and Joels, M. 2005. Mineralocorticoid receptors are indispensable for nongenomic modulation of hippocampal glutamate transmission by corticosterone. Proc. Natl. Acad. Sci. 102: 19204-19207.

Kesner, R., Bolland, B., and Dakis, M. 1993. Memory for spatial locations, motor responses, and objects: Triple dissociation among the hippocampus, caudate nucleus, and extrastriate visual cortex. Exp. Brain Res. 93: 462-470.

Kim, J., Lee, H., Han, J., and Packard, M. 2001. Amygdala is critical for stress-induced modulation of hippocampal long-term potentiation and learning. J. Neurosci. 21: 5222-5228.

Kirschbaum, C., Pirke, K.M., and Hellhammer, D.H. 1993. The 'Trier Social Stress Test' - A tool for investigating psychobiological stress responses in a laboratory setting. Neuropsychobiology 28: 76-81.

Knowlton, B.J., Mangels, J.A., and Squire, L.R. 1996. A neostriatal habit learning system in humans. Science 273: 1399-1402.

Lupien, S.J., Fiocco, A., Wan, N., Maheu, F., Lord, C., Schramek, T., and Tu, M.T. 2005. Stress hormones and human memory function across the lifespan. Psychoneuroendocrinology 30: 225-242.

Maddox, W.T. and Ashby, F.G. 2004. Dissociating explicit and procedural-learning based systems of perceptual category learning. Behav. Processes 66: 309-332.

Maguire, E.A., Burgess, N., Donnett, J.G., Frackowiak, R.S.J., Frith, C.D., and O'Keefe, J. 1998. Knowing where and getting there: A human navigation network. Science 280: 921-924. 
Matthews, D.B. and Best, P.J. 1995. Fimbria/fornix lesions facilitate the learning of a nonspatial response task. Psychon. Bull. Rev. 2: 113-116.

McDonald, R.J. and White, N.M. 1993. A triple dissociation of memory systems: Hippocampus, amygdala, and dorsal striatum. Behav. Neurosci. 107: 3-22.

McDonald, R. and White, N. 1994. Parallel information processing in the water maze: Evidence for independent memory systems involving dorsal striatum and hippocampus. Behav. Neural Biol. 61: $260-270$.

McIntyre, C.K., Gold, P.E., and Marriott, L.K. 2003. Cooperation between memory systems: Acetycholin release in the amygdala correlates positively with performance on a hippocampus-dependent task. Behav. Neurosci. 117: 320-326.

Mishkin, M. and Petri, H.L. 1984. Memories and habits. Some implications for the analysis of learning and retention. In Neuropsychology of learning and memory (eds. L. Squire and $\mathrm{N}$. Butters), pp. 287-296. Guilford Press, New York.

Nadel, L. 1991. The hippocampus and space revisited. Hippocampus 1: 221-229.

Nadel, L., Payne, J.D., and Jacobs, W.J. 2002. The relationship between episodic memory and context: Clues from memory errors made while under stress. Physiol. Res. 51: S3-S11.

Oitzl, M.S. and de Kloet, E.R. 1992. Selective corticosteroid antagonists modulate specific aspects of spatial orientation learning. Behav. Neurosci. 106: 62-71.

O'Keefe, J. and Nadel, L. 1978. The hippocampus as a cognitive map. Clarendon Press, Oxford.

Packard, M.G. 1999. Glutamate infused posttraining into the hippocampus or caudate-putamen differentially strengthens place and response learning. Proc. Natl. Acad. Sci. 96: 12881-12886.

Packard, M.G. and Knowlton, B.J. 2002. Learning and memory functions of the basal ganglia. Annu. Rev. Neurosci. 25: 563-593.

Packard, M.G. and Teather, L.A. 1998. Amygdala modulation of multiple memory systems: Hippocampus and caudate-putamen. Neurobiol. Learn. Mem. 69: 163-203.

Packard, M. and Wingard, J.C. 2004. Amygdala and "emotional" modulation of the relative use of multiple memory systems. Neurobiol. Learn. Mem. 82: 243-252.

Packard, M.G., Hirsh, R., and White, N.M. 1989. Differential effects of fornix and caudate nucleus lesions on two radial maze tasks: Evidence for multiple memory systems. J. Neurosci. 9: 1465-1472.

Packard, M.G., Cahill, L., and McGaugh, J.L. 1994. Amygdala modulation of hippocampal-dependent and caudate nucleus-dependent memory processes. Proc. Natl. Acad. Sci. 91: 8477-8481.

Poldrack, R.A., Clark, J., Paré-Blagoev, E.J., Shohamy, D., Creso Moyano, J., Myers, C., and Gluck, M.A. 2001. Interactive memory systems in the human brain. Nature 414: 546-550.

Roozendaal, B., Okuda, S., de Quervain, D.J., and McGaugh, J.L. 2006. Glucocorticoids interact with emotion-induced noradrenergic activation in influencing different memory functions. Neuroscience 138: 901-910.

Schommer, N.C., Hellhammer, D.H, and Kirschbaum, C. 2003. Dissociation between reactivity of the hypothalamus-pituitary adrenal axis and the sympathetic-adrenal-medullary system to repeated psychosocial stress. Psychosom. Med. 65: 450-460.

Schroeder, J., Wingard, J., and Packard, M. 2002. Post-training reversible inactivation of hippocampus reveals interference between memory systems. Hippocampus 12: 280-284.

Shors, T.J., Weiss, C., and Thompson, R.F. 1992. Stress-induced facilitation of classical conditioning. Science 257: 537-539.

Simon, H.A. 1982. Models of bounded rationality. MIT Press, Cambridge, MA.

Sprengelmeyer, R., Canavan, A.G., Lange, H.W., and Homberg, V. 1995. Associative learning in degenerative neostriatal disorders: Contrasts in explicit and implicit remembering between Parkinson's and Huntington's diseases. Mov. Disord. 10: 51-65.

Squire, L.R. 1994. Declarative and nondeclarative memory: Multiple brain systems supporting learning and memory. In Memory systems 1994 (ed. E. Tulving), pp. 203-232. MIT Press, Cambridge, MA.

Teng, E., Stefanacci, L., Squire, L.R., and Zola, S.M. 2000. Contrasting effects on discrimination learning after hippocampal lesions and conjoint hippocampal-caudate lesions in monkeys. J. Neurosci. 20: $3853-3863$.

Voermans, N.C., Petersson, K.M., Daudey, L., Weber, B., van Spaendonck, K.P., Kremer, H.P.H., and Fernandez, G. 2004. Interaction between the human hippocampus and the caudate nucleus during route recognition. Neuron 43: 427-435.

White, N.M. 1997. Mnemonic functions of the basal ganglia. Curr. Opin. Neurobiol. 7: 164-169.

White, N.M. and McDonald, R.J. 2002. Multiple parallel memory systems in the brain of the rat. Neurobiol. Learn. Mem. 77: 125-184.

Received September 22, 2006; accepted in revised form November 14, 2006.

\section{Learning \& Memory}

www.learnmem.org 


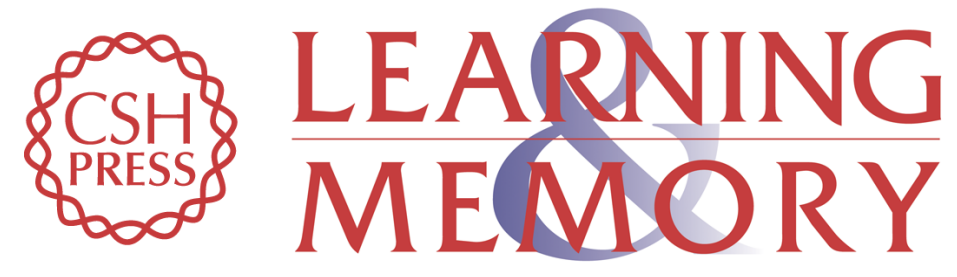

\section{Stress modulates the use of spatial versus stimulus-response learning strategies in humans}

Lars Schwabe, Melly S. Oitzl, Christine Philippsen, et al.

Learn. Mem. 2007, 14:

Access the most recent version at doi:10.1101//m.435807

References This article cites 44 articles, 14 of which can be accessed free at: http://learnmem.cshlp.org/content/14/1-2/109.full.html\#ref-list-1

License

Email Alerting Receive free email alerts when new articles cite this article - sign up in the box at the Service top right corner of the article or click here. 